\title{
El debate sobre el pensamiento computacional en educación
}

\section{(The discussion on computational thinking in education)}

\author{
Jordi Adell Segura \\ María Ángeles Llopis Nebot \\ Francesc M. Esteve Mon \\ María Gracia Valdeolivas Novella \\ Universitat Jaume I, UJI (España)
}

DOI: http://dx.doi.org/10.5944/ried.22.1.22303

\section{Como referenciar este artículo:}

Adell, J. S., Llopis, M. A. N., Esteve, M. F. M., y Valdeolivas, N. M. G. (2019).

El debate sobre el pensamiento computacional en educación. RIED.

Revista Iberoamericana de Educación a Distancia, 22(1), pp. 171-186. doi:

http://dx.doi.org/10.5944/ried.22.1.22303

\section{Resumen}

En los últimos años, un creciente número de países ha introducido el pensamiento computacional en el currículo de la educación obligatoria. En este texto, en primer lugar, analizamos brevemente algunos de los problemas que genera la ausencia de una definición consensuada de pensamiento computacional y un marco conceptual que guíe su integración en el currículo de las diversas etapas, la relación con otras disciplinas y con la competencia digital, su evaluación, la formación el profesorado, etc. En segundo lugar, se describen algunos elementos del proceso seguido en España para promover su inclusión en el currículo, un ejemplo de "política rápida" similar al de otros países, caracterizado por la compresión temporal y la fusión indiscernible de intereses públicos y privados. Finalmente, se sugiere que el discurso sobre el pensamiento computacional en educación evita el debate sobre cómo contribuye a los fines de la educación obligatoria y sobre sus asunciones previas, y sus implicaciones políticas y económicas.

Palabras clave: programa de estudios; tecnologías de la información y de la comunicación; contenido de la educación. 


\title{
J. Adell Segura; M. Á. Llopis Nebot; F. M. Esteve Mon; M. G. Valdeolivas Novella
}

EL DEBATE SOBRE EL PENSAMIENTO COMPUTACIONAL EN EDUCACIÓN

\begin{abstract}
In recent years, a growing number of countries have introduced computational thinking into the curriculum of compulsory education. In this text, firstly, we briefly analyze some of the problems generated by the absence of a consensus definition of computational thinking and a conceptual framework that guides its integration in the curriculum of the various stages, the relationship with other disciplines and with the digital competence, its evaluation, teacher training, etc. Secondly, some elements of the process followed in Spain to promote its inclusion in the curriculum are described, an example of "fast policy" similar to that of other countries, characterized by temporary compression and the indiscernible fusion of public and private interests. Finally, it is suggested that the discourse on computational thinking in education avoids the debate about how it contributes to the ends of compulsory education and about its previous assumptions and its political and economic implications.
\end{abstract}

Keywords: curriculum; information and communication technologies; content of education.

En los últimos años, el pensamiento computacional (PC en lo sucesivo) ha irrumpido con fuerza en el debate sobre el currículo de la educación obligatoria de numerosos países de nuestro entorno. En el informe del Joint Research Center de la Unión Europea (Bocconi, Chioccariello, Dettori, Ferrari y Engelhardt, 2016) que ha analizado dichas políticas en la Unión Europea y otros países se afirma que dos grandes tendencias emergen como justificación de la necesidad de su integración. Por una parte, el desarrollo de habilidades de PC en niños y jóvenes debe facilitar que "puedan pensar de manera diferente, expresarse a través de una variedad de medios, resolver problemas del mundo real y analizar temas cotidianos desde una perspectiva diferente" (Bocconi et al., 2016, p. 25). Por otra, su integración es necesaria "para impulsar el crecimiento económico, cubrir puestos de trabajo TIC y prepararse para futuros empleos" (Bocconi et al., 2016, p. 25). Es evidente que representan no solo visiones diversas del PC, sino dos maneras completamente diferentes de concebir los fines y el sentido de la educación obligatoria.

Sin embargo, pese a que los gobiernos de numerosos países han decidido que es necesario desarrollar el PC (por alguna de las razones citadas) y que la mejor manera es incorporarlo desde edades tempranas en la educación obligatoria, en este artículo pretendemos demostrar que es necesario un mayor debate, así como más investigación educativa sobre aspectos esenciales de estas iniciativas. Para ello argumentaremos que la falta de acuerdo entre los expertos sobre la definición, estructura y componentes del PC hace muy complicados aspectos como su enseñanza y evaluación válida y fiable, la inclusión de su didáctica en la formación inicial y permanente del profesorado, el establecimiento de criterios para su integración en el currículo bien como asignatura (optativa u obligatoria) en secundaria, bien 
integrado en los contenidos de las áreas STEM o $\mathrm{STEAM}^{1}$ en primaria o como actividad extracurricular. Otro aspecto problemático que requiere acuerdos es su falta de articulación con la competencia digital, una de las competencias básicas para el aprendizaje a lo largo de la vida de la Unión Europea que, en su última formulación, incluye la "programación" dentro de la "creación de contenidos digitales". El modelo de estrategia utilizada por sus defensores para promover su rápida integración curricular ha sido también objeto de críticas.

Finalmente, trataremos la situación actual en España, donde el reciente cambio político ha detenido, quizá solo momentáneamente, los planes para su implantación.

\section{LA ELUSIVA DEFINICIÓN DEL PENSAMIENTO COMPUTACIONAL}

La mayor parte de las publicaciones sitúan el origen del concepto actual de PC en una columna de opinión de Jeannette Wing publicada en el número de marzo de 2006 de la revista Communications of the ACM. En dicha publicación sostenía Wing que:

[El PC]...implica resolver problemas, diseñar sistemas y comprender el comportamiento humano, basándose en los conceptos fundamentales de la ciencia de la computación. El pensamiento computacional incluye una amplia variedad de herramientas mentales que reflejan la amplitud del campo de la computación... [además] representa una actitud y unas habilidades universales que todos los individuos, no sólo los científicos computacionales, deberían aprender y usar" (2006, p. 33).

Es decir, Wing lo definió como un conjunto de habilidades y destrezas ("herramientas mentales"), habituales en los profesionales de las ciencias de la computación, pero que todos los seres humanos deberían poseer y utilizar para "resolver problemas", "diseñar sistemas" y, sorprendentemente, "comprender el comportamiento humano". Por tanto, el PC debería formar parte de la educación de todo ser humano.

La columna de Wing tuvo un gran eco entre los profesionales de la computación. En el año 2008 se realizaron dos seminarios sobre PC en el seno del National Research Council (NRC) de los EE.UU. para explorar su naturalezay las implicaciones cognitivas y educativas en los que “... en conjunto, llama la atención la diversidad de visiones en muchos aspectos del pensamiento computacional así como en su definición" (Committee for the Workshops on Computational Thinking. National Research Council, 2011, p. viii). Tras diversos debates la International Society for Technology in Education (ISTE) y la Computer Science Teachers Association (CSTA) (2011) decidieron elaborar una definición operacional a fin de promover su inclusión en la educación obligatoria de los EE.UU. (véase tabla 1, columna 1). 
En la tabla 1, ampliación de la publicada por Denning (2017) y complementaria a la de Bocconi et al. (2016), se recogen algunas de las definiciones y marcos conceptuales más utilizados en la investigación sobre el PC y en sus formulaciones "curriculares", pero hay más matizaciones y propuestas (véase Aho, 2012; Angeli et al., 2016; Barr y Stephenson, 2011; Committee for the Workshops on Computational Thinking. National Research Council, 2010; Grover y Pea, 2013; Lee et al., 2011; Selby y Woollard, 2013; The Royal Society, 2012; Wing, 2011 o Zapata-Ros, 2015)"container-title":"Educational Technology \& Society","page":"47-57","volume" :"19","issue":"3","abstract":"Adding computer science as a separate school subject to the core K-6 curriculum is a complex issue with educational challenges. The authors herein address two of these challenges: (1. De hecho, el intento de alcanzar una definición consensuada del PC forma un notorio subconjunto de las publicaciones sobre el PC, con intentos recientes como el de Rich y Langton, (2016), que utilizan la técnica Delphi para intentar alcanzar, sin mucho éxito, cierto nivel de consenso entre un grupo de expertos, o los extensos análisis de la literatura de Corradini, Lodi y Nardelli (2017) o Haseski, Ilic y Tugtekin (2018).

La falta de acuerdo en la definición del PC es evidente desde hace tiempo. Voogt, Fisser, Good, Mishra y Yadav (2015) propusieron "olvidar" las definiciones basadas en la determinación del conjunto de cualidades que forman el "núcleo duro" del PC, aquello ineludible que lo caracteriza, y el conjunto de cualidades "periféricas" o menos cruciales que lo rodean y adoptar una perspectiva en la que se ponga el énfasis en lo "posible" y no en lo "necesario". Denning (2017, p. 34) ha criticado las definiciones del PC derivadas de la de Wing porque, por un lado, ignoran la rica historia de las ciencias de la computación y, por otro, yerran al pretender incluir los usos de la informática en todos los campos de conocimiento. El resultado es que se han hecho afirmaciones vagas y promesas exageradas sobre la utilidad del PC para solucionar cualquier tipo de problemas (recordemos la pretensión de "comprender el comportamiento humano" de Wing).

Una visión más "afilada" del PC es la de Denning (2017), basada en ideas de Aho (2012) sobre la importancia e historicidad de las abstracciones que suponen los "modelos computacionales" (como la clásica máquina de Turing). Las redes neuronales, las máquinas de reducción lógica, el aprendizaje profundo en inteligencia artificial o las analíticas de datos son ejemplos de modelos computacionales actuales. La computación es un proceso que se define en términos de un modelo computacional y, por tanto, el pensamiento computacional es el proceso de pensamiento por el que se formulan problemas de tal manera que sus soluciones puedan ser representadas como pasos computacionales y algoritmos dentro de un modelo computacional dado. Cuando se diseña un algoritmo, lo que se diseña es una manera de controlar cualquier máquina que implemente dicho modelo en orden a que ésta produzca el efecto deseado en el mundo (Denning, 2017, p. 35). ¿Es posible que las definiciones actuales del PC se basen únicamente en un modelo computacional ampliado de programación orientada a objetos? 
Por otro lado, ¿es necesario que todo el mundo posea la habilidad y la actitud de pensar computacionalmente en la era digital? Una afirmación habitual, tanto en la literatura académica como en informes de asociaciones profesionales o documentos de política educativa, es que cualquier persona necesitará esta capacidad para vivir, trabajar, aprender, comunicarse o participar como ciudadano o ciudadana de pleno derecho en la sociedad de la información. En el informe del Joint Research Center (JRC) de la Unión Europea, Bocconi et al. (2016) concluyen “... la integración del pensamiento computacional en el aprendizaje formal e informal supone una tendencia creciente y muy interesante en Europa y más allá de ella, por su potencial para la educación de una nueva generación de niños con una comprensión mucho más profunda de nuestro mundo" (p. 48).

Sin embargo, es cuestionable que todo profesional actual o futuro que use ordenadores o cualquier tipo de tecnologías de procesamiento de la información necesite diseñar algoritmos o programar, más allá de pequeños scripts que modifiquen alguna funcionalidad básica del programa. Muchas profesiones, actuales y futuras, no necesitarán programar ordenadores ni se enfrentarán al tipo de problemas solucionables mediante el pensamiento computacional. Algunas críticas, no obstante, van más lejos. Easterbrook (2014), por ejemplo, ha afirmado que, al postularse como solución universal de cualquier tipo de problemas, el PC es inherentemente reduccionista dado que existen problemas que por definición no tienen soluciones computacionales, notablemente aquellos para los que no puede definirse de antemano las características que debe poseer la solución óptima y que requieren buen juicio o acuerdo entre las partes (es decir, juicios de valor, dilemas éticos, cambio social, decisiones políticas, etc.). La perversión del PC, es decir, la aplicación del PC como estrategia de resolución de cualquier problema, está cercana al "solucionismo tecnológico" denunciado por Morozov (2013), esto es, la creencia de que la tecnología es la solución a cualquier problema humano y social, incluyendo los creados por la propia tecnología.

\section{PENSAMIENTO COMPUTACIONAL Y COMPETENCIA DIGITAL}

La relación entre el PC y la competencia digital es un tema complejo y no exento de espacios de confrontación. Todavía hay escasas publicaciones en las que se explore dicha relación en profundidad, quizá porque el concepto de competencia digital es netamente europeo y en el mundo anglosajón se utilice habitualmente el concepto de alfabetización digital, similar solo en parte. Por otra parte, existen una serie de zonas de intersección y solapamiento entre las competencias digital, informacional y mediática y el PC. Algunos autores han destacado la "superioridad" del PC (entendido como el desarrollo de habilidades generalizables de solución de problemas y el fomento de la creatividad) frente a la competencia digital (reducida a la adquisición de habilidades operacionales básicas de usuario de la tecnología). En el marco de la competencia digital de los ciudadanos de la UE, DigComp 2.1 (Carretero, Vuorikari 
y Punie, 2017) aparece "programación" dentro del apartado dedicado a la creación de contenidos digitales. Esta visión del PC como parte de la "creación de materiales" digitales se refuerza en la reciente descripción de la competencia digital que figura en la Recomendación del Consejo de 22 de mayo de 2018, relativa a las competencias clave para el aprendizaje permanente ${ }^{2}$, en la que se actualizan las definiciones de las competencias clave europeas de 2006 y se afirma:

Las personas deben ser capaces de utilizar las tecnologías digitales como apoyo a su ciudadanía activa y su inclusión social, la colaboración con otros y la creatividad para alcanzar objetivos personales, sociales o comerciales. Las capacidades incluyen la habilidad de utilizar, acceder, filtrar, evaluar, crear, programar y compartir contenidos digitales (DOUE C 189/01, 4.6.2018, p. C 189/10).

\section{PENSAMIENTO COMPUTACIONAL Y CURRÍCULO}

El posicionamiento del PC en el currículo es otro tema a debate. Una opción evidente, adoptada en diversos países es incluir una asignatura independiente en la educación secundaria (Bocconi et al., 2016), optativa, como en diversas comunidades autónomas españolas, u obligatoria. Sin embargo, gran parte del movimiento por el PC (por ejemplo, la propia Wing (2006) defiende la integración de sus elementos clave en otras áreas y asignaturas. El objetivo no es enseñar a pensar a todo el mundo como un informático, sino como médicos, arquitectos o abogados, etc. que comprendan cómo usar la computación para solucionar los problemas de sus profesiones y disciplinas y formular nuevas cuestiones que puedan ser exploradas de modo fructífero con dichas herramientas conceptuales (Hemmendinger, 2010). Barr y Stephenson (2011), Lu y Fletcher (2009) o Sengupta, Kinnebrew, Basu, Biswas y Clark (2013), entre otros, nos ofrecen ejemplos de cómo se podría integrar el PC en otras asignaturas o áreas de conocimiento.

Una tercera vía, defendida por algunos autores, no considera necesaria la integración curricular del PCy aboga por modelos extraescolares tipo Computer Club. En los últimos tiempos han proliferado en nuestro país las actividades extraescolares relacionadas con la robótica y el PC organizadas desde los propios centros o como negocio por iniciativa privada, presenciales, semipresenciales u online. La propia Unión Europea organiza eventos online financiados por empresas tecnológicas que contribuyen a alimentar en la comunidad educativa la sensación de la importancia y la urgencia de incluir en el currículo el $\mathrm{PC}^{3}$.

Existe un amplio consenso entre los expertos en que desarrollar el PC no es enseñar a programar ordenadores, pero que programar ayuda a desarrollar y a poner en acción muchas de las destrezas del PC. A pesar de ello, en la literatura suele reducirse a la enseñanza y el aprendizaje de lenguajes visuales (por ejemplo, Scratch). Pese a aportaciones sumamente interesantes, todavía no existe una 
"didáctica del PC" y la formación inicial y permanente del profesorado en este tema apenas ha empezado a investigarse (Adell, Esteve, Llopis y Valdeolivas, 2017; González, Estebanell y Peracaula, 2018; Yadav, Mayfield, Zhou, Hambrusch y Korb, 2014; Yadav, Stephenson y Hong, 2017).

Otro ejemplo de los problemas que causa la indefinición del PC es en la investigación sobre robótica educativa. ¿Contribuye la programación de robots educativos sencillos al desarrollo del PC en niños y adolescentes? En los últimos años hemos asistido a una proliferación de este tipo de actividades en educación infantil, primaria, secundaria y en contextos no formales, y a la creación de un creciente mercado de productos y servicios. Pero en una reciente revisión de la literatura, Ioannou y Makridou (2018), además de hallar solo nueve publicaciones que relacionan PC y robótica educativa, concluyen que las evidencias sobre “...el avance del pensamiento computacional como una habilidad más compleja (incluyendo descomposición, abstracción, algoritmos, depuración) fue poco clara” (sec. 3.6 Learning outcomes). Entre las recomendaciones finales a los investigadores sugieren: "ponerse de acuerdo" en una definición operacional del PC, crear instrumentos de evaluación del PC, investigar la orquestación en el aula de la robótica educativa y trabajar hacia un marco conceptual práctico para el desarrollo del PC a través de la robótica. 
Tabla 1. Diferentes marcos conceptuales del pensamiento computacional

\begin{tabular}{|c|c|c|c|c|c|}
\hline $\begin{array}{l}\text { Concepts of } \\
\text { Computational } \\
\text { Thinking } \\
\text { (International } \\
\text { Society for } \\
\text { Technology in } \\
\text { Education (ISTE) } \\
\text { y Computer } \\
\text { Science Teachers } \\
\text { Association (CSTA), } \\
\text { 2011) }\end{array}$ & $\begin{array}{l}\text { New frameworks for } \\
\text { studying and assessing } \\
\text { the development of } \\
\text { computational thinking } \\
\text { (Brennan y Resnick, 2012) }\end{array}$ & $\begin{array}{l}\text { Computing at } \\
\text { School } \\
\text { Concepts of } \\
\text { Computational } \\
\text { Thinking } \\
\text { (Csizmadia et al., } \\
\text { 2015) }\end{array}$ & $\begin{array}{l}\text { ISTE Standards } \\
\text { for Students. } \\
\text { Computational } \\
\text { Thinking } \\
\text { (ISTE, 2016) }\end{array}$ & \begin{tabular}{|} 
Conceptos y \\
procesos con alto \\
nivel de consenso \\
entre expertos \\
(Delphi) \\
(Rich y Langton, \\
2016)
\end{tabular} & $\begin{array}{c}\text { Aspectos } \\
\text { comunes en } \\
\text { una revisión } \\
\text { de literatura } \\
\text { seleccionada } \\
\text { (Corradini et al., } \\
\text { 2017) }\end{array}$ \\
\hline $\begin{array}{l}\text { Formular problemas } \\
\text { de solución } \\
\text { computacional } \\
\text { Organizar } \\
\text { logicamente y } \\
\text { analizar datos } \\
\text { Abstracciones, } \\
\text { incluyendo modelos } \\
\text { y simulaciones } \\
\text { Pensamiento } \\
\text { algorítmico } \\
\text { Evaluación de } \\
\text { la eficiencia y } \\
\text { corrección } \\
\text { Generalización y } \\
\text { transferencia a otros } \\
\text { dominios } \\
\text { Apoyado por: } \\
\text { disposiciones } \\
\text { como confianza } \\
\text { para tratar la } \\
\text { complejidad, } \\
\text { persistencia } \\
\text { en problemas } \\
\text { difíciles, tolerancia } \\
\text { a la ambigüedad, } \\
\text { problemas abiertos, } \\
\text { comunicación y } \\
\text { colaboración. }\end{array}$ & $\begin{array}{l}\text { Conceptos } \\
\text { computacionales } \\
\text { Secuencias } \\
\text { Ciclos } \\
\text { Eventos } \\
\text { Paralelismo } \\
\text { Condicionales } \\
\text { Operadores } \\
\text { Datos } \\
\text { Prácticas } \\
\text { computacionales } \\
\text { Ser incremental e iterativo } \\
\text { Ensayar y depurar } \\
\text { Reusar y remezclar } \\
\text { Abstraer y modularizar } \\
\text { Perspectivascomputacio- } \\
\text { nales } \\
\text { Expresar } \\
\text { Conectar } \\
\text { Preguntar }\end{array}$ & $\begin{array}{l}\text { Computación } \\
\text { Razonamiento } \\
\text { lógico } \\
\text { Pensamiento } \\
\text { algorítmico } \\
\text { Descomposición } \\
\text { Generalización } \\
\text { Patrones } \\
\text { Abstracción } \\
\text { Representación } \\
\text { Evaluación } \\
\text { Apoyado por: } \\
\text { técnicas de } \\
\text { reflexión, } \\
\text { codificación, } \\
\text { diseño, análisis y } \\
\text { aplicación }\end{array}$ & $\begin{array}{l}\text { Aprovechar el poder } \\
\text { de los métodos } \\
\text { tecnológicos para } \\
\text { desarrollar y probar } \\
\text { soluciones } \\
\text { Recolectar datos } \\
\text { Analizar datos } \\
\text { Representar datos } \\
\text { Descomposición } \\
\text { Abstracción } \\
\text { Algoritmos } \\
\text { Automatización } \\
\text { Pruebas } \\
\text { Paralelización } \\
\text { Simulación } \\
\text { Apoyado } \\
\text { por: aprendiz } \\
\text { empoderado, } \\
\text { ciudadano digital, } \\
\text { constructor del } \\
\text { conocimiento, } \\
\text { diseñador, } \\
\text { comunicador, } \\
\text { colaborador }\end{array}$ & $\begin{array}{l}\text { Conceptos } \\
\text { Lógica condicional } \\
\text { Eficiencia } \\
\text { Hashing (funciones } \\
\text { resumen) } \\
\text { Iteradores } \\
\text { Paralelización } \\
\text { Segmentación } \\
\text { Recursión } \\
\text { Bucles } \\
\text { Variables } \\
\text { Funciones } \\
\text { Matrices } \\
\text { Operadores } \\
\text { Gestión de eventos } \\
\text { Procesos } \\
\text { Comunicación } \\
\text { Depuración } \\
\text { Solución de } \\
\text { problemas en grupo } \\
\text { Negociación } \\
\text { Organización de } \\
\text { datos } \\
\text { Descomposición de } \\
\text { problemas }\end{array}$ & $\begin{array}{l}\text { Procesos } \\
\text { mentales: } \\
\text { Pensamiento } \\
\text { algoritmico } \\
\text { Pensamiento lógico } \\
\text { Descomposición de } \\
\text { problemas } \\
\text { Abstracción } \\
\text { Reconocimiento de } \\
\text { patrones } \\
\text { Generalización } \\
\text { Métodos: } \\
\text { Automatización } \\
\text { Recolección, análisis } \\
\text { y representación de } \\
\text { datos } \\
\text { Paralelización/ } \\
\text { Simulación } \\
\text { Evaluación/ } \\
\text { Programación } \\
\text { Prácticas: } \\
\text { Experimentar, } \\
\text { iterar, retocar/ } \\
\text { Probary ydepurar } \\
\text { /Reutilizar y } \\
\text { mezclar } \\
\text { Habilidades } \\
\text { transversales: } \\
\text { Crear/Comunicar } \\
\text { y colaborar/ } \\
\text { Reflexionar, } \\
\text { aprender, meta- } \\
\text { reflexión/Tolerancia } \\
\text { a la ambigüedad }\end{array}$ \\
\hline
\end{tabular}




\section{EL PENSAMIENTO COMPUTACIONAL EN EL CURRÍCULO DE LA EDUCACIÓN OBLIGATORIA EN ESPAÑA}

El proceso de introducción del PC en el currículo de la educación obligatoria en diversos países (Inglaterra, Suecia y Australia) es un excelente ejemplo, según Williamson, Bergviken Rensfeldt, Player-Koro y Selwyn (2018), de lo que algunos autores (Ball, Junemann y Santori, 2017; Peck y Theodore, 2015, por ejemplo) han denominado "movilidades políticas" o "políticas rápidas". Las movilidades políticas se definen por:

...la profundización de la conexión transnacional en la que los experimentos de política local existen en relación a políticas similares cercanas y lejanas, modelos itinerantes y diseños tecnocráticos, y una serie de redes financieras, técnicas, sociales y simbólicas que invariablemente funcionan a través de centros de poder y persuasión (Peck y Theodore, 2015, p. xxxi).

Las políticas rápidas se caracterizan por la "compresión temporal" en la toma de decisiones (aunque no por su "adelgazamiento jerárquico"), el desarrollo acelerado de programas, la aplicación de modelos de "buenas prácticas", las decisiones de grupos de expertos, la justificación de la competencia "internacional" en un mundo globalizado, la traducción variable al contexto, etc. Es curioso comparar las recomendaciones políticas sobre la integración del PC en el currículo de la educación obligatoria que propone el informe del Joint Research Centre de la Unión Europea (Bocconi et al., 2016, p. 49 y ss.) o el discurso "oficial" sobre cómo deben acometerse las "políticas educativas digitales" en Europa (Conrads, Rasmussen, Winters, Geniet y Langer, 2017) con los análisis de Williamson et al. (2018) sobre lo ocurrido realmente en países como Inglaterra, Suecia y Australia: son dos modelos completamente opuestos de implementar políticas educativas.

En este sentido, España parece haber optado, hasta la fecha, por la "política rápida”. Un par de documentos son esenciales para comprender las estrategias utilizadas y los roles desempeñados por diversos agentes e instancias.

El 22 de abril de 2016 la Fundación Española para la Ciencia y la Tecnología (FECYT) del Ministerio de Economía y Competitividad del Gobierno de España, Google y Everis publicaron un informe titulado Educación en Ciencias de la Computación en España $2015^{4}$ con el objetivo de "analizar la situación actual de la enseñanza de Ciencias de la Computación (CC) en España para niños y niñas de entre 6 y 16 años" y "propone(r) una serie de recomendaciones para la introducción, expansión y mejora de la enseñanza de esta materia en el corto y medio plazo" (p. 3). Una de las recomendaciones finales del informe fue "Establecer un marco de consenso entre los agentes clave sobre la hoja de ruta a seguir para introducir las $\mathrm{CC}$ en el currículo educativo, tanto en Educación Primaria como Secundaria” (p. 56). Aunque no se indique directamente que las empresas del sector formen parte 
de los "agentes clave" en la elaboración de dicha hoja de ruta, en la explicación de dicha propuesta se afirma que "La integración de las CC en el currículo debe ser apoyada por las Administraciones Públicas y la industria, proveyendo contenidos y materiales formativos, así como coordinando comunidades para compartir las mejores prácticas" (p. 56).

En enero de 2018 el Ministerio de Educación, Cultura y Deporte (MEDC) del Gobierno de España publicó el informe Programación, robótica y pensamiento computacional en el aula. Situación en España, enero $2018^{5}$ en el que se describe la situación actual de dichos contenidos en el currículo oficial nacional y en el de diversas comunidades autónomas. También se recogen diversas iniciativas desarrolladas por universidades, empresas o la sociedad civil. El informe fue elaborado por el Instituto Nacional de Tecnologías Educativas y de Formación del Profesorado (INTEF) y el Centro Nacional de Innovación e Investigación Educativa (CNIIE), ambos pertenecientes al MECD, con la participación de representantes de las consejerías y departamentos de educación de doce comunidades autónomas, de empresas tecnológicas (BQ, Microsoft, Telefónica y, nuevamente, Everis y Google), empresas educativas (Institución Educativa SEK), universidades (UNED, Girona y Rey Juan Carlos), asociaciones profesionales (Sociedad Científica Informática Española ${ }^{6}$ y entidades que desarrollan actividades pioneras en este campo (Programamos).

En el informe se describe la situación actual de la programación, la robótica y el pensamiento computacional en el currículo básico y de diferentes comunidades autónomas y diversas iniciativas no oficiales, lideradas desde empresas, universidades o la sociedad civil. También se incluye una sección titulada "Opinión del profesorado español" que recoge los resultados de una encuesta online dirigida a los docentes y contestada por 351 personas en la que, entre otros temas, se valoran positivamente (aunque sin excesivo entusiasmo: 7,34 sobre 10) los resultados de aprendizaje de los alumnos en experiencias sobre programación y robótica. En las conclusiones del informe no se ofrecen recomendaciones concretas a la administración, tan solo se destaca que, a pesar de que apenas existen contenidos relacionados con la programación, la robótica y el pensamiento computacional en el currículo básico actual, varias comunidades autónomas han comenzado a incluir dichos contenidos en diferentes asignaturas, especialmente en educación secundaria, pero se trata de asignaturas optativas y por tanto es posible completar la secundaria sin haber recibido formación en este ámbito. Por otra parte, solo un tercio de los docentes que contestaron la encuesta declararon haber recibido formación sobre estos temas, siendo el resto autodidactas. Por último, el informe reconoce que es necesaria más investigación sobre estos temas en nuestro país.

Pero en la nota de prensa sobre dicho informe publicada en la web del $\mathrm{MECD}^{7}$ se afirma:

El grupo de trabajo que ha elaborado el informe va a continuar su acción en los próximos meses elaborando una propuesta normativa para incorporar la 
programación, la robótica y el pensamiento computacional al currículo en todos los niveles educativos, que se espera que esté publicada antes del verano.

Cuando se redactan estas líneas (junio de 2018), una reciente moción de censura ha provocado un cambio de gobierno en España y no es posible predecir si seguirá en vigor la agenda para la introducción de la programación, la robótica y el pensamiento computacional en el currículo oficial de todos los niveles de la educación básica.

\section{CONCLUSIONES: PENSAMIENTO COMPUTACIONAL, IDEOLOGÍA Y TECNOLOGÍA, UN DEBATE NECESARIO}

El PC ha irrumpido con fuerza en los sistemas educativos de un creciente número de países de nuestro entorno en los últimos años, que lo han incluido en el currículo de la educación obligatoria o están considerando su integración. Sin embargo, por un lado, existe una evidente falta de consenso entre los expertos a nivel mundial sobre un marco conceptual que lo defina con precisión y que describa sus principales componentes. La consecuencia es que no disponemos de criterios sobre cómo enseñarlo en las distintas etapas educativas, cómo integrarlo dentro de las disciplinas actuales o sobre si es preferible que sea una actividad extraescolar, ni en cómo evaluarlo o formar al profesorado de los distintos niveles.

Por otra parte, las razones por las que el PC debe formar parte de la educación de todos los ciudadanos se alinean con dos visiones muy diferentes del fin de la educación obligatoria: la que la reduce a la adquisición de habilidades supuestamente demandadas por el mercado de trabajo y la que pretende desarrollar la capacidad expresiva y comunicativa de niños y adolescentes. Entre las razones que justifican su inclusión en el currículo está notablemente ausente la necesidad de dotar a los ciudadanos y ciudadanas de los conceptos, habilidades y actitudes precisas para desarrollar una competencia digital crítica que les permita afrontar su proyecto de vida en una sociedad en la que en las últimas décadas se han producido cambios radicales en la economía, la política y la cultura. Comprender qué es un algoritmo requiere también comprender por qué nuestra vida y nuestras relaciones, por ejemplo, con la administración y el poder, están cada vez más mediatizadas por sistemas algorítmicos, en qué tipo de supuestos se basan dichos sistemas, qué consecuencias tienen en nuestras vidas y qué derechos debemos reivindicar. Enseñar a programar no es una decisión neutral, científica y objetiva. Es una decisión política y las propuestas habituales, dirigidas únicamente a la capacitación operacional y técnica, refuerzan la visión neoliberal de la educación como únicamente una inversión en capital humano.

Los agentes de las políticas curriculares rápidas parecen responder más a los intereses y deseos de nuevos y poderosos jugadores en la determinación del currículo, las empresas de tecnología que hoy dominan la economía mundial, que a las necesidades reales de los ciudadanos y ciudadanas de la sociedad digital. En 
este sentido, cierta manera de entender el PC en educación no solo forma parte de la ideología hegemónica que emana de Silicon Valley, sino que uno de sus objetivos ocultos sería bajar los salarios de los profesionales de la computación (Tarnof, 2017). No solo es necesaria más investigación para rellenar nuestros huecos de conocimiento sobre el PC, también es necesario un amplio debate social sobre qué futuro queremos contribuir a construir.

\section{NOTAS}

1. STEM o STEAM es el acrónimo en inglés de las asignaturas o contenidos educativos relacionados con Ciencia, Tecnología, Ingeniería (Arte, en ocasiones) y Matemáticas.

2. Recomendación del Consejo, de 22 de mayo de 2018, relativa a las competencias clave para el aprendizaje permanente (DOUE C 189/01, 4.6.2018) https://eur-lex.europa. eu/legal-content/EN/TXT/?qid=1530889560212\&uri=CONSIL:ST 54642018 INIT

3. Véase, por ejemplo, la página web de la EU Code Week http://codeweek.eu

4. Accesible en la web de la FECYT: https://www.fecyt.es/es/publicacion/educacion-delas-ciencias-de-la-computacion-en-espana

5. Dicho informe puede consultarse en http://code.educalab.es/wp-content/ uploads/2017/09/Pensamiento-Computacional-Fase-1-Informe-sobre-la-situaciónen-España.pdf

6. La Sociedad Científica Informática Española (SCIE) también ha publicado un informe sobre la enseñanza preuniversitaria de la informática en que se reconoce que el PC “... es un término poco preciso y que no permite centrar fácilmente el debate educativo" (p. 11). Accesible en http://www.scie.es/wp-content/uploads/2018/o7/informe-sciecoddii-2018.pdf

7. Nota de prensa: "El Ministerio de Educación, Cultura y Deporte presenta el informe "Programación, robótica y pensamiento computacional en el aula. Situación en España, enero 2018”. 20 de febrero de 2018. https://www.mecd.gob.es/prensa-mecd/ actualidad/2018/02/20180220-robot.html

\section{REFERENCIAS}

Adell, J., Esteve, F., Llopis, M. Á., y Valdeolivas, M. G. (2017). El pensamiento computacional en la formación inicial del profesorado de infantil y primaria. En V. Abella García, V. Ausín Villaverde y V. Delgado Benito (Eds.), Actas de las XXV Jornadas Universitarias de Tecnología Educativa JUTE 2017. Aulas y Tecnología Educativa en evolución (pp. 151-158). Burgos, 21, 22 y 23 de junio de 2017. Recuperado de http://www3.ubu.es/ jute2017/wp-content/uploads/2018/03/ JUTE2017-LIBRO DE ACTAS.pdf

Aho, A. V. (2012). Computation and Computational Thinking. The Computer Journal, 55(7), 832-835. doi: https://doi. org/10.1093/comjnl/bxso74

Angeli, C., Voogt, J., Fluck, A., Webb, M., Cox, M., Malyn-Smith, J., y Zagani, J. (2016). A K-6 Computational Thinking Curriculum Framework: Implication for Teacher Knowledge. Educational Technology \& Society, 19(3), 47-57. 
Recuperado de https://www.jstor.org/ stable/jeductechsoci.19.3.47

Ball, S. J., Junemann, C., y Santori, D. (2017). Edu.net: globalisation and education policy mobility. London; New York, NY: Routledge.

Barr, V., y Stephenson, C. (2011). Bringing computational thinking to $\mathrm{K}-12$ : What is involved and what is the role of the computer science education community? ACM Inroads, 2(1), 48-54. doi: https:// doi.org/10.1145/1929887.1929905

Bocconi, S., Chioccariello, A., Dettori, G., Ferrari, A., y Engelhardt, K. (2016). Developing computational thinking in compulsory education implications for policy and practice.Sevilla: Joint Research Centre. doi: http://doi. org/10.2791/792158

Brennan, K., y Resnick, M. (2012). New frameworks for studying and assessing the development of computational thinking. Proceedings of the 2012 Annual Meeting of the American Educational Research Association, 1-25. Recuperado de https:// web.media.mit.edu/ kbrennan/files/ Brennan Resnick AERA2012 CT.pdf

Carretero, S., Vuorikari, R., y Punie, Y. (2017). DigComp 2.1: The Digital Competence Framework for Citizens with eight proficiency levels and examples of use (No. EUR 28558 EN). Recuperado de http://doi.org/10.2760/38842

Committee for the Workshops on Computational Thinking. National Research Council (2010). Report of a Workshop on The Scope and Nature of Computational Thinking. Washington, D.C.: The National Academies Press. doi: https://doi.org/10.17226/12840

Committee for the Workshops on Computational Thinking. National Research Council (2011). Report of a Workshop on the Pedagogical Aspects of Computational Thinking. Washington, D.C.: The National Academies Press. Recuperado de https://www.nap.edu/ catalog/13170/report-of-a-workshopon-the-pedagogical-aspects-ofcomputational-thinking

Conrads, J., Rasmussen, M., Winters, N., Geniet, A., y Langer, L. (2017). Digital education policies in Europe and beyond key design principles for more effective policies. En C. Redecker, P. Kampylis, M. Bacigalupo y Y. Punie, (Eds.), Luxembourg: Publications Office of the European Union. Recuperado de http:// doi.org/10.2760/462941

Corradini, I., Lodi, M., y Nardelli, E. (2017). Conceptions and misconceptions about computational thinking among Italian primary school teachers. En Proceedings of the 2017 ACM Conference on International Computing Education Research (pp. 136-144). ACM.

Csizmadia, A., Curzon, P., Dorling, M., Humphreys, S., Ng, T., Selby, C., y Woollard, J. (2015). Computational Thinking. A guide for Teachers. Computing At School. Recuperado de http://community.computingatschool. org.uk/files/6695/original.pdf

Denning, P. J. (2017). Remaining trouble spots with computational thinking. Communications of the ACM, 6o(6), 3339. doi: https://doi.org/10.1145/2998438 Easterbrook, S. (2014). From Computational Thinking to Systems Thinking: A conceptual toolkit for sustainability computing. Proceedings of the 2nd International Conference on Information and Communication Technologies for Sustainability (ICT4S'2014), Stockholm, Sweden, (pp. 24-27) August, 2014. Recuperado de http://www.cs.toronto. edu/ sme/papers/2014/EasterbrookICT4S-2014.pdf

González, J., Estebanell, M., y Peracaula, M. (2018). ¿Robots o programación? El concepto de Pensamiento Computacional $\mathrm{y}$ los futuros maestros = Robotics or Coding? The Concept of Computational 
Thinking in Pre-service Teachers. EKS, 19(2), 29-45.

Grover, S., y Pea, R. (2013). Computational Thinking in K-12: A Review of the State of the Field. Educational Researcher, 42(1), 38-43. doi: https://doi. org/10.3102/0013189X12463051

Haseski, H. I., Ilic, U., y Tugtekin, U. (2018). Defining a New 21st Century SkillComputational Thinking: Concepts and Trends. International Education Studies, 11(4), 29. doi: https://doi.org/10.5539/ ies.v11n4p29

Hemmendinger,D.(2010).Apleaformodesty. ACM Inroads, 1(2), 4-7. doi: https://doi. org/10.1145/1805724.1805725

International Society for Technology in Education (ISTE) y Computer Science Teachers Association (CSTA). (2011). Operational Definition of Computational Thinking for K-12 Education. Recuperado de http://www.iste.org/ docs/ct-documents/computationalthinking-operational-definition-flyer. pdf?sfvrsn $=2$

Ioannou, A., y Makridou, E. (2018). Exploring the potentials of educational robotics in the development of computational thinking: A summary of current research and practical proposal for future work. Education and Information Technologies. doi: https://doi.org/10.1007/s10639018-9729-Z

ISTE. (2016). ISTE Standards for Students. Washington DC: International Society for Technology in Education. Recuperado de https://www.iste.org/standards/forstudents

Lee, I., Martin, F., Denner, J., Coulter, B., Allan, W., Erickson, J., ... Werner, L. (2011). Computational thinking for youth in practice. $A C M$ Inroads, 2(1), 32-37. doi: https://doi. org/10.1145/1929887.1929902

Lu, J. J., y Fletcher, G. H. L. (2009). Thinking about computational thinking. SIGCSE Bulletin Inroads,
41(1), 260-264. doi: https://doi. org/10.1145/1539024.1508959

Peck, J., y Theodore, N. (2015). Fast policy: experimental statecraft at the thresholds of neoliberalism. Minneapolis: University of Minnesota Press.

Rich, P. J., y Langton, M. B. (2016). Computational Thinking: Toward a Unifying Definition. En J. M. Spector, D. Ifenthaler, D. G. Sampson, \& P. Isaias (Eds.), Competencies in Teaching, Learning and Educational Leadership in the Digital Age (pp. 229-242). Cham: Springer International Publishing. doi: https://doi.org/10.1007/978-3-31930295-9 14

Selby, C., y Woollard, J. (2013). Computational thinking: the developing definition. Presentado en SIGCSE 2014, 5-8 March, Atlanta GA. Recuperado de https://eprints.soton.ac.uk/356481/1/ Selby Woollard bg soton eprints.pdf

Sengupta, P., Kinnebrew, J. S., Basu, S., Biswas, G., y Clark, D. (2013). Integrating computational thinking with $\mathrm{K}-12$ science education using agent-based computation: A theoretical framework. Education and Information Technologies, 18(2), 351-380. doi: https://doi.org/10.1007/ s10639-012-9240-X

Tarnof, B. (2017, 21 de septiembre). Tech's push to teach coding isn't about kids' success - it's about cutting wages. The Guardian. Recuperado de https://www. theguardian.com/technology/2017/ sep/21/coding-education-teachingsilicon-valley-wageshttps://www. theguardian.com/technology/2017/ sep/21/coding-education-teachingsilicon-valley-wages

The Royal Society. (2012). Shutdown or restart? The way forward for computing in UK schools. London.

Voogt, J., Fisser, P., Good, J., Mishra, P., y Yadav, A. (2015). Computational thinking in compulsory education: Towards an agenda for research and practice. 
Education and Information Technologies, 2O(4), 715-728. doi: https://doi. org/10.1007/s10639-015-9412-6

Williamson, B. (2015). Political computational thinking: policy networks, digital governance and 'learning to code'. Critical Policy Studies, 1-20. doi: https:// doi.org/10.1080/19460171.2015.1052003

Williamson, B., Bergviken Rensfeldt, A., Player-Koro, C., y Selwyn, N. (2018). Education recoded: policy mobilities in the international 'learning to code' agenda. Journal of Education Policy. doi: https://doi.org/10.1080/02680939.2018 .1476735

Wing, J. M. (2006). Computational thinking. Communications of the ACM, 49(3), 33-35. doi: https://doi. org/10.1145/1118178.1118215

Wing, J. M. (2011). Computational thinking. En 2011 IEEE Symposium on
Visual Languages and Human-Centric Computing (VL/HCC 2011) (pp. 3-3). IEEE. doi: https://doi.org/10.1109/ VLHCC.2011.6070404

Yadav, A., Mayfield, C., Zhou, N., Hambrusch, S., y Korb, J. T. (2014). Computational Thinking in Elementary and Secondary Teacher Education. TOCE, 14(1), 1-16. doi: https://doi.org/10.1145/2576872

Yadav, A., Stephenson, C. y Hong, H. (2017). Computational thinking for teacher education. Communications of the ACM, 6o(4), 55-62. doi: https://doi. org/10.1145/2994591

Zapata-Ros, M. (2015). Pensamiento computacional: Una nueva alfabetización digital. RED. Revista de Educación a Distancia, (46). Recuperado de http:// www.um.es/ead/red/46

\section{PERFIL ACADÉMICO Y PROFESIONAL DE LOS AUTORES}

Jordi Adell Segura. Profesor Titular del Departamento de Pedagogía y Didáctica de las Ciencias Sociales, la Lengua y la Literatura de la Universitat Jaume I (Castellón, España). Director del grupo de investigación GREAT (Enseñanza, Aprendizaje y Tecnología) de la Universitat Jaume I y del Centro de Educación y Nuevas Tecnologías de la misma universidad. Sus líneas de investigación actuales incluyen el análisis de la competencia digital en la formación inicial docente y el impacto de la tecnología en la enseñanza y el aprendizaje universitarios. E-mail: jordi@uji.es

María Ángeles Llopis Nebot. Profesora Asociada del Departamento de Pedagogía y Didáctica de las Ciencias Sociales, la Lengua y la Literatura de la Universitat Jaume I (Castellón, España). Investigadora en el Grupo de Enseñanza, Aprendizaje y Tecnología (GREAT) de la Universitat Jaume I. Sus líneas de investigación se centran en el análisis de la competencia digital y la formación inicial docente.

E-mail: mallopis@uji.es 
Francesc Esteve Mon. Profesor Ayudante Doctor del Departamento de Pedagogía y Didáctica de las Ciencias Sociales, la Lengua y la Literatura de la Universitat Jaume I (Castellón, España). Miembro del grupo de investigación GREAT (Enseñanza, Aprendizaje y Tecnología) de la Universitat Jaume I. Colaborador del grupo de investigación ARGET (Applied Research Group in Education and Technology) y de la Cátedra UNESCO de Gestión y Política Universitaria. Centra su investigación en el análisis de la competencia digital y la formación inicial docente. E-mail: festeve@uji.es

María Gracia Valdeolivas Novella. Profesora Asociada del Departamento de Pedagogía y Didáctica de las Ciencias Sociales, la Lengua y la Literatura de la Universitat Jaume I (Castellón, España). Miembro del grupo de investigación GREAT (Enseñanza, Aprendizaje y Tecnología) de la Universitat Jaume I. Sus líneas de investigación se centran en el análisis de la competencia digital y la formación inicial docente. Profesora en Colegio La Magdalena (Castellón, España).

E-mail:valdeoli@uji.es

Dirección:

Facultat de Ciències Humanes i Socials, Universitat Jaume I

Avda. Sos Baynat s/n, 12071a

Castelló de la Plana (España)

Fecha de recepción del artículo: 07/07/2018

Fecha de aceptación del artículo: 27/07/2018

Fecha de aprobación para maquetación: 06/10/2018 